H. Kim

Nagoya Math. J.

Vol. 150 (1998), 85-94

\title{
MODULI SPACES OF STABLE VECTOR BUNDLES ON ENRIQUES SURFACES
}

\author{
HOIL KIM
}

\begin{abstract}
We show that the image of the moduli space of stable bundles on an Enriques surface by the pull back map is a Lagrangian subvariety in the moduli space of stable bundles, which is a symplectic variety, on the covering K3 surface. We also describe singularities and some other features of it.
\end{abstract}

\section{$\S 0$. Introduction}

Moduli spaces of stable vector bundles on algebraic surfaces have been described by several authors. Vector bundles on rational surfaces ([Ba], $[\mathrm{Hu}])$, ruled surfaces $([\mathrm{Br}],[\mathrm{Q}]), \mathrm{K} 3$ surfaces $([\mathrm{Mu} 1,2],[\mathrm{Ty} 1,2])$, elliptic surfaces $([\mathrm{F}],[\mathrm{O}, \mathrm{V}])$ and some surfaces of general type $([\mathrm{Bh}],[\mathrm{D}, \mathrm{K}])$ have been studied. In this paper we want to study the moduli spaces of stable bundles on Enriques surfaces. Every Enriques surface has a K3 surface as a universal covering space.

Mukai ([Mu1]) showed that the moduli space of stable vector bundles on any K3 surface has a symplectic structure. We will describe the moduli spaces of stable bundles on Enriques surfaces with relation to those on the corresponding $\mathrm{K} 3$ surfaces.

THEOREM. (1) The image of the moduli space of stable bundles on an Enriques surface by the pull back map is a Lagrangian subvariety, exactly the fixed locus of the induced involution, in the moduli space of stable bundles, which is a symplectic variety, on the covering $\mathrm{K} 3$ surface.

(2) The singularities in the moduli space $M$ of stable bundles on an Enriques surface are the images of finitely many union of the moduli spaces of the moduli spaces of stable bundles on the $\mathrm{K} 3$ surface and the dimension of singular locus is at most $\frac{1}{2}(\operatorname{dim} M+3)$ (big codimensional singularity).

(3) The pull back map is two to one from the smooth locus of $M$ to the moduli space of stable bundles on the K3 surface, with no branch locus.

Received August 16, 1994. 
This paper is based on some part of my thesis, where we proved a weaker form of the theorem and has been improved during the stay at Bayreuth University and Max-Planck-Institut in Bonn. I thank to Professor I. Dolgachev for suggesting this problem and guiding and to two institutions for good hospitality with financial support. Takemoto's old result was very important to our work, which I did not notice before. I thank Professor C. Okonek for indicating that paper to me and for some other helpful discussions. I thank Professors, Ono and Borovoi for a lemma in chapter 2 and D. Huybrecht for a good comment in the proof of the theorem and discussions.

\section{$\S 1$. Preliminaries}

1. An Enriques surface $X$ is a minimal algebraic surface whose canonical divisor $K_{X} \nsim 0$, but $2 K_{X} \sim 0$, where $\sim$ denotes the linear equivalence.

2. Every Enriques surface has an elliptic structure over $\mathbf{P}^{1}$. It has exactly two multiple fibres of multiplicity 2 , say them $F_{A}, F_{B}$. Then the canonical divisor can be expressed as a difference of two multiple fibres, that is $K_{X} \sim F_{A}-F_{B}$.

3. The fundamental group of any Enriques surface is $\mathbf{Z}_{2}$, so that the universal covering space is a K3 surface. Let the quotient map be $\pi$. That is an etale covering with respect to $K_{X}$. So $\pi_{*}\left(O_{\bar{X}}\right) \cong O_{X} \oplus K_{X}, \pi^{*}\left(K_{X}\right) \cong$ $O_{\bar{X}}$.

4. An Enriques surface $X$ is called nodal if there exists a smooth rational curve $R$. (In this case $R^{2}=-2$.) Otherwise, it is called unnodal. In the 10 dimensional moduli space of Enriques surfaces, a generic one is unnodal, while the nodal ones form a 9 dimensional subvariety ([C,D]).

5. A nodal cycle $N$ on an Enriques, or a K3 surface is a positive 1-cycle such that $h^{1}\left(O_{N}\right)=0$. This is a tree of smooth rational curves ([Ar]).

6. We define the slope of $E$ with respect to some ample divisor $H$, denoted by $\mu_{H}(E)$, as $\left(c_{1}(E) \cdot H\right) / \operatorname{rank}(E)$. A vector bundle $E$ is called $H$ (-semi)-stable if for every subsheaf $F$, with $0<\operatorname{rank}(F)<\operatorname{rank}(E)$,

$$
\mu_{H}(F)<(\leq) \mu_{H}(E)
$$

There exists a moduli space of stable vector bundles which is a quasiprojective algebraic variety.

7. Let $X$ be an Enriques surface (K3 surface). Then the map $c_{1}$ : Pic $X \rightarrow H^{2}(X, Z)$ is an isomorphism (injective). So, we identify $\operatorname{Pic} X$ with its image. 
Now we fix the notations.

$X$ is an Enriques surface and its universal covering space, which is a K3 surface, is denoted by $\bar{X}$ and the quotient map from $\bar{X}$ to $X$ is $\pi$. Let $M_{X, H}\left(r, c_{1}, c_{2}\right)$ (resp. $\left.M_{\bar{X}, \pi^{*} H}\left(r, c_{1}, c_{2}\right)\right)$ be the moduli space of stable vector bundles on $X$ (resp. $\bar{X}$ ) with respect to $H$ (resp. $\pi^{*} H$ ), where $r$ is the rank of the bundles and $c_{i}$ is the assignment of the $i$-th Chern class.

We denote by $i$ the involution on $\bar{X}$ compatible to $\pi$ and by $i^{*}$ the induced involution on $M_{\bar{X}}$. We mean $K_{X}$ by $K$ and $E \otimes L$ by $E(L)$, where $L$ is a line bundle and $E$ is a vector bundle.

\section{$\S 2$. General structure theorem}

Here we interpret the results of Takemoto $[\mathrm{Ta}]$ in our Enriques surface $X$ and the covering K3 surface $\bar{X}$.

TheOREm. [Ta] (1) If a $\pi^{*} H$-stable bundle $F$ on $\bar{X}$ is not isomorphic to $\pi^{*} E$ for any bundle $E$ on $X$, then $\pi_{*}(F)$ is $H$-stable. IfF is $\pi^{*} H$ semi-stable, then $\pi_{*} F$ is $H$-semi-stable.

(2) If a simple bundle $E$ on $X$ is isomorphic to $E(K)$, then there exists a simple bundle $F$ on $X$ such that $\pi_{*}(F) \cong E$.

Next we introduce the result of Mukai on the moduli spaces of stable bundles on K3 surfaces.

TheOREM. [Mu1] The moduli space $M$ of stable bundles on a K3 surface $S$ is smooth and there is a line bundle $L=O_{M}$ and a skew-symmetric bilinear form $B: T M \times T M \rightarrow L$ such that $B \otimes k([F])$ is nondegenerate and canonically isomorphic to the natural pairing $\operatorname{Ext}^{1}(F, F) \times \operatorname{Ext}^{1}(F, F) \rightarrow$ $\operatorname{Ext}^{2}(F, F)$ for any stable bundle $F$.

Let us begin with a lemma.

Lemma. Let $X$ be an Enriques surface and $\bar{X}$, be the universal covering space of $X$ and $F$ be a simple vector bundle on $\bar{X}$ such that $F \cong i^{*} F$. Then there exists a bundle $E$ on $X$ such that $\pi^{*} E \cong F$.

Proof. It suffices to prove that there exists a map $f: F \rightarrow F$, over the involution such that $f^{2}=\mathrm{id}$. For a given isomorphism $h: F \rightarrow i^{*} F$, let $g$ be a composition of $h$ followed by the natural map $j: i^{*} F \rightarrow F$. Then $g$ is a map from $F$ to $F$ over the involution and $g^{2}=\lambda I d$ for some $\lambda \neq 0$ in $\mathbf{C}$. Let $f$ be $(\lambda)^{-1 / 2} g$. Then, $f$ satisfies the required property. 
Remark. This can be genralized to any bundle $F$ whose endomorphism is $M_{r}(\mathbf{C})$, where $r$ is the rank of $E$.

Before going into the main theorem, we recall the formula for the dimension of $M=M_{X}\left(r, c_{1}, c_{2}\right)$, the moduli space of stable bundles on an Enriques surface $X$, and the dimension of the tangent space $T_{E} M$ at $E \in M$.

$$
\begin{aligned}
\operatorname{dim}_{E} M & \geq 2 r c_{2}-(r-1) c_{1}^{2}-r^{2}+1 \\
\operatorname{dim} T_{E} M & =2 r C_{2}-(r-1) c_{1}^{2}-r^{2}+1+h^{2}(\text { End } E) .
\end{aligned}
$$

Here $h^{2}($ End $E)=0$ if $E \not ⿻ E(K)$ and 1 if $E \cong E(K)$. This comes from the fact that for any non-trivial homomorphism between two stable bundles with the same slope is an isomorphism. $([\mathrm{O}, \mathrm{S}, \mathrm{S}])$ The right hand side of the above inequality is called is the expected dimension of $M$.

Then we state our main result.

THEOREM. Let $\bar{X}$ be a K3 surface which is the universal covering space of an Enriques surface $X$ and $M_{X},\left(\right.$ resp. $\left.M_{\bar{X}}\right)$ be a moduli space of stable vector bundles on $X$ (resp. $\bar{X})$ ( see $\S 1)$.

(1) Then, $M_{X}$ is singular at $E$ if and only if $E \cong E(K)$ except the case that $E$ belongs to a 0 -dimensional component (exceptional bundle) or a two dimensional component, where every bundle $E$ satisfies that $E \cong E(K)$. The singular locus of $M_{X}$ is a union of the images by $\pi$ of finitely many different components of $M_{\bar{X}}^{0}$ with possibly different Chern classes on $\bar{X}$, where $M_{\bar{X}}^{0}=\left\{F \in M_{\bar{X}} \mid F \neq i^{*} F\right\}$ and its dimension is $\leq \frac{1}{2}\left(\operatorname{dim} M_{X}+\right.$ 3). So, $M_{X}$ is generically smooth. In particular, if the rank is odd, it is everywhere smooth, and if the rank $=2$ then it can have only finitely many isolated singular points.

(2) The pull back map $\pi^{*}$ from $M_{X}^{0}$ to $M_{\bar{X}}$ is two to one with no branch, where $M_{X}^{0}=\left\{E \in M_{X} \mid E \nRightarrow E(K)\right\}$.

(3) The image of $M_{X}^{0}$ by $\pi^{*}$ is a Lagrangian subvariety in $M_{\bar{X}}$ and is equal to the fixed locus by involution $i^{*}$.

Proof of (1). If $E$ is a singular point in $M_{X}$ then $E \cong E(K)$, so that $E \cong \pi_{*} F$ for some stable bundle $F$ on $X$. ([Ta], or $\S 1$ ) So, the rank is an even number, say $2 k$. Then $\pi^{*} E \cong F \oplus i^{*} F$. Let the Chern polynomial of $F$ be $1+c_{1}(F) t+c_{2}(F) t^{2}$. Then, that of $i^{*} F$ is $1+\left(i^{*} c_{1}(F)\right) t+c_{2}(F) t^{2}$. So, we have

$$
\begin{aligned}
\pi^{*} c_{1}(E) & =c_{1}(F)+i^{*} c_{1}(F), \\
2 c_{2}(E)=\pi^{*} c_{2}(E) & =c_{1}(F) \cdot i^{*} c_{1}(F)+2 c_{2}(F) .
\end{aligned}
$$


Here $c_{1}(F) \cdot \pi^{*} H=i^{*} c_{1}(F) \cdot \pi^{*} H$ since $\pi^{*} H \sim i^{*}\left(\pi^{*} H\right)$. This implies that $\left(c_{1}(F)-i^{*} c_{1}(F)\right)^{2} \leq 0$ by the Hodge Index theorem (the equality holds if and only if $\left.c_{1}(F)=i^{*} c_{1}(F)\right)$. Now we can find a relationship between the dimensions of $M_{X}\left(2 k, c_{1}(E), c_{2}(E)\right)$, and $M_{\bar{X}}\left(k, c_{1}(F), c_{2}(F)\right)$.

$$
\begin{aligned}
\operatorname{dim} M_{X}= & 4 k c_{2}(E)-(2 k-1) c_{1}^{2}(E)-4 k^{2}+1 \\
= & 2 k\left(c_{1}(F) \cdot i^{*} c_{1}(F)\right)+4 k c_{2}(F) \\
& -(2 k-1)\left(c_{1}^{2}(F)+c_{1}(F) \cdot i^{*} c_{1}(F)\right)-4 k^{2}+1 \\
= & 2\left(2 k c_{2}(F)-(k-1) c_{1}^{2}(F)-2 k^{2}+2\right) \\
& +\left(c_{1}(F) \cdot i^{*} c_{1}(F)-c_{1}^{2}(F)\right)-3 .
\end{aligned}
$$

(This is a computation when $M_{X}$ is of expected dimension. For the component not of expected dimension, where $E \cong E(K)$, we must add by 1 .) However we know that

$$
\left(c_{1}(F) \cdot i^{*} c_{1}(F)-c_{1}^{2}(F)\right) \geq 0,
$$

where the equality holds if and only if $c_{1}(F)=i^{*} c_{1}(F)$. So, we can conclude that

$$
\operatorname{dim} M_{\bar{X}}\left(k, c_{1}(F), c_{2}(F)\right) \leq \frac{1}{2}\left(\operatorname{dim} M_{X}\left(2 k, c_{1}(E), c_{2}(E)\right)+3\right)
$$

where the equality holds if and only if $c_{1}(F)=i^{*} c_{1}(F)$. (We have $\operatorname{dim} M_{\bar{X}} \leq$ $\frac{1}{2}\left(\operatorname{dim} M_{X}+2\right)$, the equality holds if and only if $c_{1}(F)=i^{*} c_{1}(F)$ for the component not of expected dimension.)

From the above formula, we see that

$$
c_{1}(F)^{2}-c_{1}(F) \cdot i^{*} c_{1}(F) \geq-\operatorname{dim} M_{X}-3=B,
$$

so that,

$$
2 B \leq\left(c_{1}(F)-i^{*} c_{1}(F)\right)^{2} \leq 0 .
$$

So, there can be only finitely many numbers for $\left(c_{1}(F)-i^{*} c_{1}(F)\right)^{2}$ and for a fixed value there can be only finitely many choices for $c_{1}(F)$, since $\left(\pi^{*} H\right)^{\perp}$ is a negative definite lattice. However, $\pi_{*} F$ is stable if and only if $F \not i^{*} F$. So the singular locus of $M_{X}$ is a finitely many union of direct images of $M_{\bar{X}}^{0}$. Here the map $\pi_{*}$ from these $M_{\bar{X}}$ to the singular locus of $M_{X}$ is 2 to 1 with no branch. In fact $\pi_{*}(F) \cong \pi_{*}(G)$ implies that $\pi^{*}\left(\pi_{*}(F)\right) \cong \pi^{*}\left(\pi_{*}(G)\right)$. This means that $F \oplus i^{*} F \cong G \oplus i^{*} G$. So

$$
\begin{aligned}
& \operatorname{Hom}(F, G) \oplus \operatorname{Hom}\left(F, i^{*} G\right) \oplus \operatorname{Hom}\left(i^{*} F, G\right) \oplus \operatorname{Hom}\left(i^{*} F, i^{*} G\right) \\
\cong & \operatorname{Hom}\left(F \oplus i^{*} F, G \oplus i^{*} G\right) \neq 0 .
\end{aligned}
$$


So, this forces that $F \cong G$, or $F \cong i^{*} G$. Obviously, $\pi_{*} F \cong \pi_{*}\left(i^{*} F\right)$.

If $\pi_{*}$ is 1 to 1 , then $F \cong i^{*} F$ and $\pi_{*} F$ is not stable. The singular locus is of even dimension and smooth in itself. If the rank is odd, then $M$ is everywhere smooth.

Conversely, if $E \cong E(K)$, then $E \cong \pi_{*} F$ for some $F$ on $X$. If $E$ is a smooth point in $M_{X}$, then $E \cong E(K)$ everywhere in the component $M$ containing $E$. Then $M$ is a finite union of the images of some components of $M_{\bar{X}}$ with possibly different Chern classes, which have the same dimension as $M$, call one of the components $\bar{M}$. From the previous formula, we get

$$
\operatorname{dim} M=\operatorname{dim} \bar{M} \leq \frac{1}{2}(\operatorname{dim} M+2)
$$

(Note that $E \cong E(K)$ in $M$ ). So, the possible dimension of $M$ is 0 or 2 . If $\operatorname{dim} M=2$, then for $F$ in $\bar{M}, c_{1}(F)=i^{*} c_{1}(F)$, so that $c_{1}(F)=\frac{1}{2} \pi^{*} c_{1}(E)$ and if $\operatorname{dim} M=0, M$ has a unique bundle $E$ and $\bar{M}$ has two bundles $F$ and $i^{*} F$ such that $c_{1}^{2}(F)=c_{1}(F) \cdot i^{*} c_{1}(F)-2$. If the rank is 2 , then the singular locus is the direct images of finitely many different line bundles. This completes the proof of (1).

Remark 1. In fact, the singularity of $M_{X}\left(2 k, c_{1}, c_{2}\right)$ is closely related to the singularity of the curves in the linear system of $c_{1}$ (the splitting behaviour of the divisor of $\pi^{*}\left(c_{1}\right)$ on $\bar{X}$.)

Remark 2. There can be 3 different types of components in $M_{X},(1)$ a component $M$ which has the expected dimension and is smooth everywhere, (2) a component $M$ which has the expected dimension, but has some singularity, (3) a component $M$ which has the dimension one bigger than the expected dimension (must be smooth everywhere). The singularity can exist only in the second type. The components with codimension one singularity can exist only for $\operatorname{dim} M=1,3$ or 5 .

We will give those examples.

EXAMPLE 1. The simplest example of $M_{X}$ with some singularities is $M_{X}\left(2, F_{A}, 1\right)$, where $F_{A}$ is a half fibre. Then $M_{X}\left(2, F_{A}, 1\right)=F_{B}$, another half fibre. If $F_{B}$ is singular with an ordinary double point, then the inverse image of $F_{B}$ is a union of two smooth rational curves $R_{1}, R_{2}=i^{*}\left(R_{1}\right)\left(\bar{A}_{1}\right.$ type). Then the bundle $E$ corresponding to the singularity is just $\pi_{*} O_{\bar{X}}\left(R_{1}\right)$ (or $\left.R_{2}\right)$. Note that $\operatorname{det}\left(\pi_{*} O_{\bar{X}}\left(R_{1}\right)\right) \sim F_{B}+K \sim F_{A}([\mathrm{Ha}])$. 
EXAMPLE 2. We can find many examples of the moduli space $M$ of dimension three whose singularity is a K3 surface. We can find a compact moduli space $M_{\bar{X}}\left(k, c_{1}, c_{2}\right)$ such that $c_{1}^{2}=c_{1} \cdot i^{*} c_{1}-2$ (this holds if and only if $c_{1}=N+S$, where $N$ is a nodal cycle with $N \cdot i^{*} N=0$ and $S$ is a divisor fixed by involution and $\operatorname{dim} M_{\bar{X}}=2$. There are many examples with these conditions. Then $M_{\bar{X}}$ is a K3 surface and the dimension of the corresponding $M_{X}$ is 3 and the singular locus is just the image of that $\mathrm{K} 3$ surface. (Note that $\pi_{*} M_{\bar{X}}\left(k, c_{1}, c_{2}\right)=\pi_{*} M_{\bar{X}}\left(k, i^{*} c_{1}, c_{2}\right)$ and $c_{1} \neq i^{*} c_{1}$.)

EXAMPLE 3. If we choose $M_{\bar{X}}\left(k, c_{1}, c_{2}\right)$ of dimension 4 with $c_{1}=i^{*} c_{1}$ then the image of $M_{\bar{X}}^{0}$ by $\pi_{*}$ is the singular locus of 4 dimension in the 5-dimensional space, $M_{X}$.

Proof of (2). First we show that if $E$ is $H$-stable and is not isomorphic to $E(K)$, then $\pi^{*} E$ is $\pi^{*} H$-stable, From the fact that

$$
H^{0}\left(\text { End } \pi^{*} E\right)=H^{0}(\text { End } E) \oplus H^{0}((\text { End } E)(K))=\mathbf{C},
$$

$\pi^{*} E$ is simple. $\pi^{*} E$ is also a direct sum of stable bundles with the same slope (since the pull back of an Einstein-Hermitian bundle is still EinsteinHermitian and an Einstein-Hermitian bundle is a direct sum of stable bundles with the same slope.) From these two facts $\pi^{*} E$ must be $\pi^{*} H$-stable. From the above equation we conclude also that if $E$ is isomorphic to $E(K)$, then $\pi^{*} E$ is not simple, just a direct sum of stable bundles. (In fact, $\pi^{*} E \cong F \oplus i^{*} F$, for some $F$ such that $\pi_{*} F \cong E$. ([Ta])) So, $\pi^{*}$ is well defined from $M_{X}^{0}=\left\{E \mid E \in M_{X}, E \not E(K)\right\}$ to $M_{\bar{X}} . M_{X}^{0}$ is the same as the smooth locus of $M_{X}$ except two cases as we saw in the proof of (1). Next we show that $\pi^{*}$ is 2 to 1 with no branch. If $\pi^{*} E \cong \pi^{*} E^{\prime}$, then $H^{0}\left(\pi^{*}\left(E^{*} \otimes E^{\prime}\right)\right) \neq 0$. However,

$$
H^{0}\left(\pi^{*}\left(E^{*} \otimes E^{\prime}\right)\right)=H^{0}\left(E^{*} \otimes E^{\prime}\right) \oplus H^{0}\left(E^{*} \otimes E^{\prime}(K)\right) .
$$

So, either $H^{0}\left(E^{*} \otimes E^{\prime}\right) \neq 0$, or $H^{0}\left(E^{*} \otimes E^{\prime}(K)\right) \neq 0$. The property of stability implies $E \cong E^{\prime}$, or $E \cong E^{\prime}(K)$. So, the map $\pi^{*}$ is 2 to 1 with no branch if the rank is even and 1 to 1 if the rank is odd.

Remark. (a) $\pi^{*}$ restricted to $M_{X}^{0}\left(2 k, D, c_{2}\right)$ or $M_{X}^{0}\left(2 k, D+K, c_{2}\right)$ is still 2 to 1 with no branch. In general, $M_{X}\left(2 k, D, c_{2}\right)$ is not isomorphic to $M_{X}\left(2 k, D+K, c_{2}\right)$. For example, $M_{X}\left(2, F_{A}, 1\right)=F_{B}$ is not isomorphic 
to $M_{X}\left(2, F_{B}, 1\right)=F_{A}$. If an exceptional bundle $E$ of even rank exists for $\operatorname{det}(E)=D$, then there is no exceptional bundle $E^{\prime}$ for $\operatorname{det}\left(E^{\prime}\right)=D+K$.

(b) However, $\pi_{*}$ restricted to $M_{X}\left(2 k+1, D, c_{2}\right)$ or $M_{X}\left(2 k+1, D+K, c_{2}\right)$ is 1 to 1 , so that $M_{X}\left(2 k+1, D, c_{2}\right)\left(=M_{X}\left(2 k+1, D+K, c_{2}\right)\right)$ is isomorphic to its image.

Proof of (3). First we show that the dimension of $M_{X}^{0}\left(r, c_{1}, c_{2}\right)$ is half of the dimension of $M_{\bar{X}}\left(r, \pi^{*} c_{1}, \pi^{*} c_{2}\right)$.

$$
\begin{aligned}
\operatorname{dim} M_{\bar{X}} & =2 r \pi^{*} c_{2}-(r-1)\left(\pi^{*} c_{1}\right)^{2}-2 r^{2}+2 \\
& =2\left(2 r c_{2}-(r-1) c_{1}^{2}-r^{2}+1\right)=2 \operatorname{dim} M_{X}^{0} .
\end{aligned}
$$

Next we show that the pull back of the holomorphic two form $\omega$ on $M_{\bar{X}}$ to $M_{X}^{0}$ vanishes. The proof comes easily from the following commuting diagram,

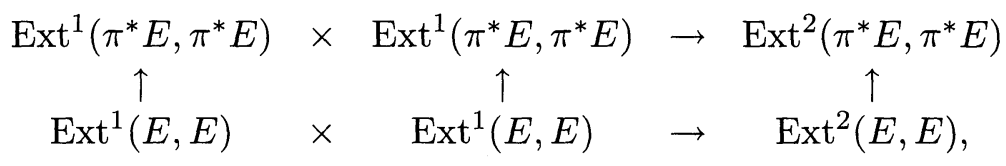

and the fact that $\operatorname{Ext}^{2}(E, E)=H^{2}($ End $E)=0$, for any $E \in M_{X}^{0}$. (In the above diagram, $\operatorname{Ext}^{1}(E, E)=T_{E} M_{X}$ and $\operatorname{Ext}^{1}\left(\pi^{*} E, \pi^{*} E\right)=T_{\pi^{*}} M_{\bar{X}}$. $)$ So, we can also conclude that the image of $M_{X}^{0}$ is a Lagrangian subvariety in $M_{\bar{X}}$. That the image of $M_{X}^{0}$ is fixed by involution $i^{*}$ is obvious. Another direction comes from the lemma easily. So the image is exactly the fixed locus by involution. This completes the proof of (3).

Remark 1. We expect that $M_{\bar{X}}$ is birational to the cotangent bundle of the image of $M_{X}^{0}$ by $\pi^{*}$.

Remark 2. We know that the dimension of $M_{X}\left(2 k+1, c_{1}, c_{2}\right)$ is even. We expect that $M_{X}\left(2 k+1, D, c_{2}\right)$ is birational to a symmetric power of some Enriques surface. In fact we know many cases that $M_{\bar{X}}$ is birational to a symmetric power of some K3 surface. In this case, the image of $M_{X}$ is just the fixed locus by involution, so that it is a symmetric power of an Enriques surface, the quotient of that K3 surface. Another example is $M_{X}\left(3, c_{1}, 3\right)$, where $X$ is a fourfold covering of $\mathbf{P}^{2}$ and $c_{1}$ is a pull back of hyperplane of $\mathbf{P}^{2}$. Then $c_{1}^{2}=4$. In this case $M$ is birational to the original Enriques surface $X$. 


\section{REFERENCES}

[Ar] M. Artin, Some numerical criteria for contractability of curves on algebraic surfaces, A. J. Math., 84 (1962), 485-496.

[Ba] W. Barth, Moduli of vector bundles on the projective plane, Invent. Math., 42 (1977), 63-91.

[Bh] U. N. Bhosle, Net of quadrics and vector bundles on a double plane, Math. Z., 192 (1986), ??-??

$[B, P, V]$ W. Barth, C. Peters and A. Van de Ven, Compact Complex Surfaces, SpringerVerlag, 1984.

[Br] E. Brosius, Rank 2 vector bundles on a ruled surface I, II, Math. Ann., 265 (1983), 155-168, Math. Ann., 266 (1983), 199-214.

[C,D] F. Cossec and I. Dolgachev, Enriques Surfaces I, Birkheuser 1989.

$[D, K]$ S. K. Donaldson and P. B. Kronheimer, The Geometry of Four Manifolds, Oxford, 1990.

[D,R] I. Dolgachev and I. Reider, On rank 2 vector bundles with $c_{1}^{2}=10$ and $c_{2}=3$ on Enriques surfaces, LNM, Springer-Verlag, 1479 (1991).

[F] R. Friedman, Rank two vector bundles over regular elliptic surfaces, Invent Math., 96 (1989), 283-332.

[Ha] R. Hartshorne, Algebraic Geometry, Springer-Verlag, 1977.

[Hu] K. Hulek, Stable rank 2 vector bundles on $\mathbf{P}^{2}$ with $c_{1}$ odd, Math. Ann., 242 (1979), 241-266.

[Ki] H. Kim, Exceptional bundles on nodal Enriques surfaces, Manuscripta Math., 82 (1994), 1-13.

[Mu1] S. Mukai, Symplectic structure of the moduli space of sheaves on an abelian or K3 surface, Invent. Math., 77 (1984), 101-116.

[Mu2] S. Mukai, On the moduli space of bundles on K3 surfaces I, Vector Bundles (Atiyah et al, ed.), Oxford Univ. Press, Bombay (1980), pp. 341-413.

[O,S,S] C. Okonek, M. Schneider and H. Spindler, Vector bundles on complex projective spaces, Progress in Math. Vol.3, Birkhauser, Boston (1980).

$[\mathrm{O}, \mathrm{V}]$ C. Okonek and A. Van de Ven, Stable bundles and differential structures on certain elliptic surfaces, Invent Math., 86 (86), 357-370.

[Ta] F. Takemoto, Stable vector bundles on algebraic surfaces II, Nagoya Math. J., 52 (1973), 173-195.

[Ty1] A. Tyurin, Cycles, curves and vector bundles on an algebraic surface, Duke Math. J., 54 (1987), 1-26.

[Ty2] A. Tyurin, Symplectic structures on the varieties of moduli of vector bundles on algebraic surfaces with $p_{g}>0$, Math. USSR Izvestiya, 33, No.1 (1989), 139-177.

[Q] Z. Qin, Moduli spaces of stable rank 2 bundles on ruled surfaces, Invent. Math., 110 (1992), 615-626. 
H. KIM

Topology and Geometry Research Center

Kyungpook National University

Taegu

Korea

hikim@bh.kyungpook.ac.kr 